\title{
Regulatory strategies to schedule threshold crossing of protein levels at a prescribed time
}

\author{
César Nieto ${ }^{1}$, Khem Raj Ghusinga ${ }^{2}$, and Abhyudai Singh ${ }^{1}$
}

\begin{abstract}
The timing of diverse cellular processes is based on the instant when the concentration of regulatory proteins crosses a critical threshold level. Hence, noise mechanisms inherent to these protein synthesis pathways drive statistical fluctuations in such events' timing. How to express proteins ensuring both the threshold crossing at a prescribed time and minimal timing fluctuations? To find this optimal strategy, we formulate a model where protein molecules are synthesized in random bursts of gene activity. The burst frequency depends on the protein level creating a feedback loop, and cellular growth dilutes protein concentration between consecutive bursts. Counterintuitively, our analysis shows that positive feedback in protein production is best for minimizing variability in thresholdcrossing times. We analytically predict the optimal feedback strength in terms of the dilution rate. As a corollary to our result, a no-feedback strategy emerges as the optimal strategy in the absence of dilution. We further consider other noise sources, such as randomness in either the initial condition or the threshold level, and find that in many cases, we need either strongly negative or positive feedback for precise scheduling for events.
\end{abstract}

\section{INTRODUCTION}

Proper timing of molecular and cellular events is critical for a wide range of cellular processes in development, decision-making, signal transduction, coordination of responses, etc. [1]-[8]. In many cases, transcriptional response drives the timing of important events whereby the event occurs upon accumulation of a regulatory protein up to a critical threshold level [2], [9]-[22]. The inherent stochastic nature of gene-expression causes cell-to-cell variability in the time evolution of the protein levels - and consequently in the timing of cellular events - even for cells with identical genetic content and the same environmental conditions [23][28]. A fundamental question of interest is to understand how cells control temporal dynamics of the underlying regulatory protein to ensure precision in event timing.

Recent works have studied precise scheduling of events for gene-expression models of varying complexity, where the timing of an event is formulated as the first-passage time (FPT) for the protein level [29]-[41]. Assuming different empirical forms for the regulation of gene-expression (e.g., feedback auto-regulation, activation/repression by an upstream component, etc.), these works search for the best model parameters that minimize the noise in timing [34][36], [38], [39], [42], [43]. In the limiting case where protein does not degrade (or dilute), any form of feedback gives

\footnotetext{
${ }^{1}$ Department of Electrical and Computer Engineering, University of Delaware, Newark, DE, USA \{cnieto, absingh\} eudel.edu

${ }^{2}$ Department of Biology, University of North Carolina at Chapel Hill, Chapel Hill, NC, USA khem@udel. edu
}

higher noise in event timing around a fixed mean time [35]. This result is robust to extrinsic disturbances [44] and several physiologically relevant variations in model parameters [35], [45], except for the case when the initial protein amount is drawn from a distribution [46]. For a protein that degrades, positive feedback from the protein level to the transcription rate suppresses noise in event timing around a fixed mean by counterpoising the effect of degradation [35], [42], [43]. These two findings suggest a relationship between the degradation rate of the protein and the strength of the positive feedback to schedule events with precision. However, the exact nature of this relationship remains to be explored.

In this work, we consider a model of gene expression based on stochastic hybrid system formalism. Protein production is assumed to occur in bursts and is modeled as a stochastic event, whereas dilution due to cell growth is modeled using a deterministic ODE [38], [47], [48]. We provide analytical results on FPT moments and compute the optimal feedback strategy that minimizes noise in FPT around a given fixed mean under different modeling assumptions. In particular, we consider a linear form of the feedback and analyze scenarios where we draw either the initial condition or the event threshold from some positivevalued distributions, representing static extrinsic noise.

\section{PREliminaries}

In this section, we formulate a model of gene-expression and describe the corresponding FPT problem. We then summarize the previous results that quantify the noise in FPT and provide optimal feedback strategies that minimize the noise.

\section{A. Model description}

Let $x(t)$ be the concentration of the protein of interest at time $t$. We assume that the event of interest occurs when $x(t)$ crosses the threshold level $X$ for the first time (Fig. 1). The FPT $T$ when the event $x$ reaches a threshold $X$ is defined formally as

$$
T:=\inf \left\{t \geq 0: x(t) \geq X \mid x(0)=x_{0}<X\right\},
$$

where $x_{0}$ is protein level at $t=0$.

We model the dynamics of $x(t)$ using a stochastic hybrid system (SHS) formalism comprising both discrete jumps and continuous dilution. In particular, we assume that $x(t)$ is produced in bursts as

$$
x(t) \mapsto x(t)+B,
$$


where the burst size, $B$, follows a positive-valued distribution. More precisely, the probability, $\mathbb{P}$, of arrival of a burst in an infinitesimal time interval $(t, t+d t)$ is given by

$$
\mathbb{P}\{x(t+d t)=i+B \mid x(t)=i\}=\frac{k(i)}{\langle B\rangle} d t .
$$

Here, we assume that the protein production rate is a function of the protein level as $k(x)$. This is motivated by investigating how different feedback mechanisms might affect the noise in event timing. In this framework, an open-loop production (no feedback) implies a constant rate $k(x)=k$, a negative feedback means that $k(x)$ decreases with $x$, and positive feedback signifies that $k(x)$ increases with $x$. We have used the mean burst size $\langle B\rangle$ as a normalization constant. Wherever needed for analytical tractability, we will assume a linear form of the feedback as $k(x)=k_{0}+k_{0} x$. Here $k_{0}$ is the basal rate of protein production, $k_{1}$ is the strength of the feedback, and $\langle B\rangle$ is the mean burst size used as a normalizing factor convenience.

In between successive bursts, the protein concentration is diluted by cell growth as

$$
d x=-\gamma x d t
$$

Here $\gamma$ denotes the dilution rate. Finally, we assume that both the initial protein level $x(0)=x_{0}$ and the threshold for the event $X$ are drawn from arbitrary positive-valued distributions.

We base this model formulation on the following key assumptions. First, the protein does not degrade but only dilutes due to cell growth. This assumption itself requires that the cell volume grows exponentially over time and that the production rate in terms of protein numbers scales with the cell volume [49]-[52]. Second, both the timescale of the promoter switching and the mRNA half-life are much smaller than the turnover rate of the protein. This allows us to approximate the randomness in the dynamics of promoter and mRNA in a burst parameter. Often, the burst size follows a geometric distribution [53], but other distributions are also possible [45], [54]-[56].

The statistics of the first-passage time (FPT) defined in Eq. (1) are analytically intractable in general. However, in a limiting case where dilution is ignored, the FPT moments were computed in previous work [35]. Next, we reproduce these results and the computation of optimal feedback strategy to minimize the noise in timing from them.

\section{B. FPT statistics in absence of dilution}

Let $\mathbb{P}(x(t)=i)=p_{i}(t)$, then in absence of dilution, i.e., $\gamma=0$, the time evolution of $p_{i}$ for Eq. (2b) is governed by the chemical master equation [57], [58]

$$
\frac{d p_{i}}{d t}=-\frac{k(i)}{\langle B\rangle} \mathbb{P}(B \geq 1) p_{i}(t)+\sum_{j=0}^{i-1} \frac{k(j)}{\langle B\rangle} \mathbb{P}(B=i-j) p_{j}(t) .
$$

To compute the statistics of the first-passage time, $T$, as defined in Eq. (1), we note that [35]

$$
\mathbb{P}(T \in(t, t+d t))=\sum_{i=0}^{X-1} \frac{k(i)}{\langle B\rangle} \mathbb{P}(B \geq X-i) p_{i}(t) d t,
$$

resulting in the following probability density function (pdf) of FPT

$$
f_{T}(t)=\sum_{i=0}^{X-1} \frac{k(i)}{\langle B\rangle} \mathbb{P}(B \geq X-i) p_{i}(t) .
$$

Intuitively, the process $x(t)$ crosses the threshold $X$ for the first time at time $t+d t$ if the protein count was equal $i$ at time $t$ and a burst of size greater than or equal to $X-i$ occurred in the next infinitesimal time interval $(t, t+d t)$.

A compact form of the FPT pdf above is

$$
f_{\tau}(t)=\mathbf{U}^{\top} \mathbf{P}(t)
$$

where

$$
\begin{aligned}
\mathbf{P}(t) & =\left[\begin{array}{llll}
p_{0}(t) & p_{1}(t) & \ldots & p_{X-1}(t)
\end{array}\right]^{\top} \\
\mathbf{U} & =\left[\begin{array}{lll}
\frac{k(0)}{\langle B\rangle} \mathbb{P}(B \geq X) & \ldots & \frac{k(X-1)}{\langle B\rangle} \mathbb{P}(B \geq 1)
\end{array}\right] .
\end{aligned}
$$

The time evolution of $\mathbf{P}(t)$ can be obtained from Eq. (3) as the dynamical system

$$
\dot{\mathbf{P}}=\mathbf{A P},
$$

whose solution is given by

$$
\mathbf{P}(t)=\exp (\mathbf{A} t) \mathbf{P}(0) .
$$

Here the triangular matrix $A$ consists of the elements

$$
a_{i, j}= \begin{cases}0, & j \geq i+1, \\ -\frac{k(i-1)}{\langle B\rangle} \times \mathbb{P}(B \geq 1), & j=i, \\ \frac{k(j-1)}{\langle B\rangle} \times \mathbb{P}(B=i-1), & j<i,\end{cases}
$$

and $\mathbf{P}(0)$ is the vector of consisting of probabilities of the initial protein count, $x_{0}$. Using (7b) in (6a) yields the following for the first-passage time probability density function

$$
f_{T}(t)=\mathbf{U}^{\top} \exp (\mathbf{A} t) \mathbf{P}(0) .
$$

We can use Eq. (8) to compute the moments of FPT as follows

$$
\begin{aligned}
\left\langle T^{m}\right\rangle & =\int_{0}^{\infty} t^{m} \mathbf{U}^{\top} \exp (\mathbf{A} t) \mathbf{P}(0) d t \\
& =\mathbf{U}^{\top}\left(\int_{0}^{\infty} t^{m} \exp (\mathbf{A} t)\right) \mathbf{P}(0) d t,
\end{aligned}
$$

where $\langle$.$\rangle denotes expectation of its argument and m$ is the order of the moment. The above integral may be written as

$$
\left\langle T^{m}\right\rangle=m !(-1)^{m+1} \mathbf{U}^{\top}\left(\mathbf{A}^{-1}\right)^{m+1} \mathbf{P}(0) .
$$

provided $\mathbf{A}$ is Hurwitz stable. This indeed the case, as each diagonal element of $\mathbf{A}$ is negative and is greater in magnitude than sum of all other elements of that column [35]. The first two moments of FPT for the special case of deterministic 
(A)

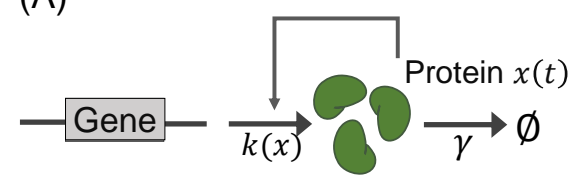

(B)

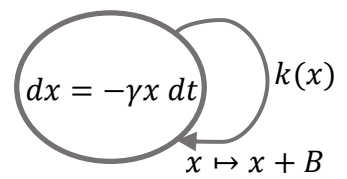

(C)

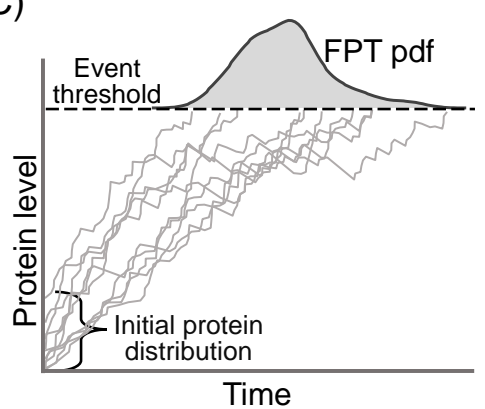

Fig. 1. Model schematic and illustration of first-passage time. (A) Gene produces protein $x(t)$ in bursts. The burst frequency depends upon the protein level as $k(x)$, creating a feedback loop. The protein degrades/dilutes through a first-order kinetics with rate constant $\gamma$. (B) A stochastic hybrid system representation of the gene-expression model, whereby the protein level increases upon burst arrival at a rate that depends upon the protein level. Protein dilution is modeled using an ordinary differential equation. (C) First-passage time for the protein level to reach a threshold. Sample trajectories represent different realizations of the protein evolution obtained using Monte Carlo simulations. The initial protein level is drawn from a distribution.

initial condition $x_{0}=0$, deterministic threshold $X$, and geometrically distributed burst size are given by:

$$
\begin{aligned}
& \langle T\rangle=\sum_{i=0}^{X-1} \frac{1}{k(i)}+\frac{\langle B\rangle}{k(0)} \\
& \langle T\rangle=\frac{2}{\langle B\rangle^{2}}\left(\frac{\tau_{0}}{k(0)}+\sum_{i=0}^{X-1} \frac{\tau_{i}}{k_{i}}\right), \tau_{i}:=\frac{1}{k_{i}}+\sum_{j=i}^{X-1} \frac{\langle B\rangle}{k_{j}} .
\end{aligned}
$$

One can use conditioning argument to compute these moments when the initial condition $x_{0} \neq 0$ and is instead drawn from a distribution [46]. Likewise, the event threshold may also be drawn from any positive-valued distribution. These scenarios are studied in section IV (Figs. 4 and 5).

\section{Optimal feedback strategy}

The problem of finding an optimal feedback strategy that minimizes the noise in timing (quantified by the coefficient of variation squared $=$ variance $/$ mean $^{2}$ ), given a fixed mean $\langle T\rangle$, is a constrained optimization problem. It yields the following analytical solution via the method of Lagrange multiplier [35]:

$$
\begin{aligned}
k(0) & =\frac{1+\langle B\rangle}{1+2\langle B\rangle} \frac{2\langle B\rangle+X}{\langle T\rangle}, \\
k(x) & =\frac{2\langle B\rangle+X}{\langle T\rangle}, \forall x=\{1,2, \ldots, X-1\} .
\end{aligned}
$$

In the limit of a small burst size, the above solution is essentially a no feedback strategy. Even for a geometrically distributed burst size, all the production rates are equal except for the first one; thus, the optimal strategy is approximately a no feedback.

\section{OPTIMAL FEEDBACK STRATEGY FOR BURSTY GENE EXPRESSION}

We next focus our attention on the case $\gamma \neq 0$ and particularly restrict ourselves to the class of linear feedback, where the burst frequency takes the form $\left(k_{0}+k_{1} x(t)\right) /\langle B\rangle$.
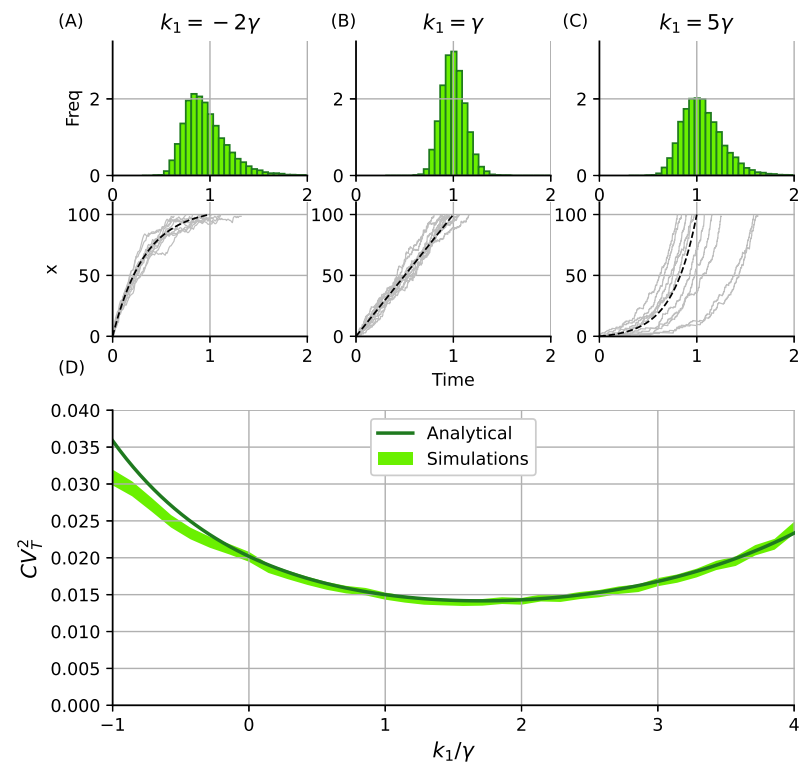

Fig. 2. FPT fluctuations for different feedback strategies with trajectories modelled using a birth death process. The feedback strategies are classified depending on the ratio between the decay rate $\gamma$ and the feedback strength $k_{1}$. (A) $k_{1}<\gamma$ (B) $k_{1}=\gamma$ (C) $k_{1}>\gamma$ Top: FPT histogram. Bottom: Some illustrative trajectories. (D) Noise in FPT measured by the squared coefficient on variation $C V_{T}^{2}$ vs the feedback strength $k_{1}$. Results of simulations using Gillespie algorithm (region width representing 95\% confidence interval calculated with ten thousand simulation replicas) are compared to the analytical expression (19). $(\gamma=1,\langle T\rangle \approx 1, x(0)=0$, $X=100$, and $\langle B\rangle=1$ with probability one). [59].

As a consequence of this linearity, the time evolution of the statistical moment of $x(t)$ can be obtained exactly via

$$
\frac{d\left\langle x^{m}\right\rangle}{d t}=\left\langle\frac{k_{0}+k_{1} x}{\langle B\rangle}\left((x+B)^{m}-x^{m}\right)-\gamma m x^{m}\right\rangle
$$

for $m \in\{1,2, \ldots\}$ [61]-[63]. Setting $m=1$ and assuming $x(0)=0$ with probability one, yields the mean concentration 

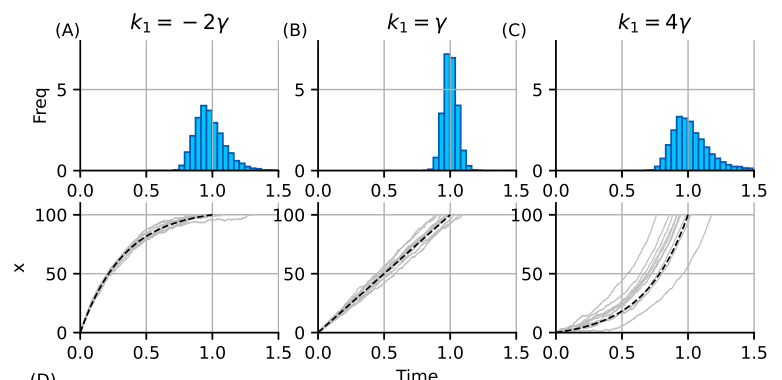

(D)

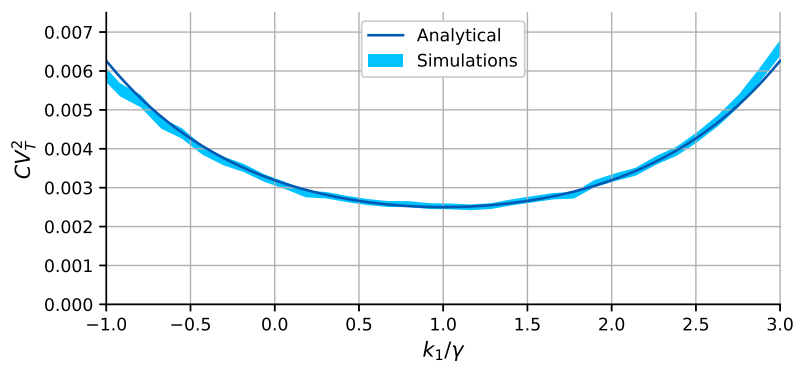

Fig. 3. FPT fluctuations for different feedback strategies with trajectories modelled using a stochastic differential equation. The feedback strategies are classified depending on the ratio between the decay rate $\gamma$ and the feedback strength $k_{1}$. (A) $k_{1}<\gamma$ (B) $k_{1}=\gamma$ (C) $k_{1}>\gamma$ Top: FPT histogram. Bottom: Some illustrative trajectories. (D) Noise in FPT measured by the squared coefficient on variation $C V_{T}^{2}$ vs the feedback strength $k_{1}$. Results of simulations using Euler-Maruyama algorithm [60] (region width representing 95\% confidence interval over ten thousand simulation replicas) are compared to the analytical expression (25). $(\gamma=1$, $\langle T\rangle \approx 1, x(0)=0, X=100, \sigma=5)$.

dynamics

$$
\begin{aligned}
\frac{d\langle x\rangle}{d t} & =k_{0}+k_{1}\langle x\rangle-\gamma\langle x\rangle \\
\Longrightarrow\langle x(t)\rangle & =\frac{k_{0}\left(1-e^{\left(-\gamma+k_{1}\right) t}\right)}{\gamma-k_{1}} .
\end{aligned}
$$

If the feedback strength $k_{1}<\gamma$ then $\lim _{t \rightarrow \infty}\langle x(t)\rangle=$ $k_{0} /\left(\gamma-k_{1}\right)$, and we assume that the critical concentration threshold $X<k_{0} /\left(\gamma-k_{1}\right)$ needed for event timing is below this steady-state mean level. In contrast, if $k_{1} \geq \gamma$ then the mean concentration grows unboundedly $\lim _{t \rightarrow \infty}\langle x(t)\rangle=$ $\infty$ over time. Considering small concentration fluctuations around the mean trajectory, the mean first passage time

$$
\langle T\rangle \approx \frac{\ln \left[\frac{k_{0}}{k_{0}+\left(k_{1}-\gamma\right) X}\right]}{\gamma-k_{1}}
$$

can be obtained by solving $\langle x(t)\rangle=X$. Recall that we would like to obtain the optimal feedback strategy (i.e., the value of $k_{1}$ ) that minimizes the noise in $T$ for a given fixed mean FPT $\langle T\rangle$. Having an approximate analytical formula for $\langle T\rangle$ is quite useful in that regard as it provides the corresponding value of $k_{0}$

$$
k_{0}=\frac{\left(k_{1}-\gamma\right) X}{e^{\left(k_{1}-\gamma\right)\langle T\rangle}-1}
$$

that is needed to ensure a fixed $\langle T\rangle$ as we vary $k_{1}$ to explore different feedbacks.
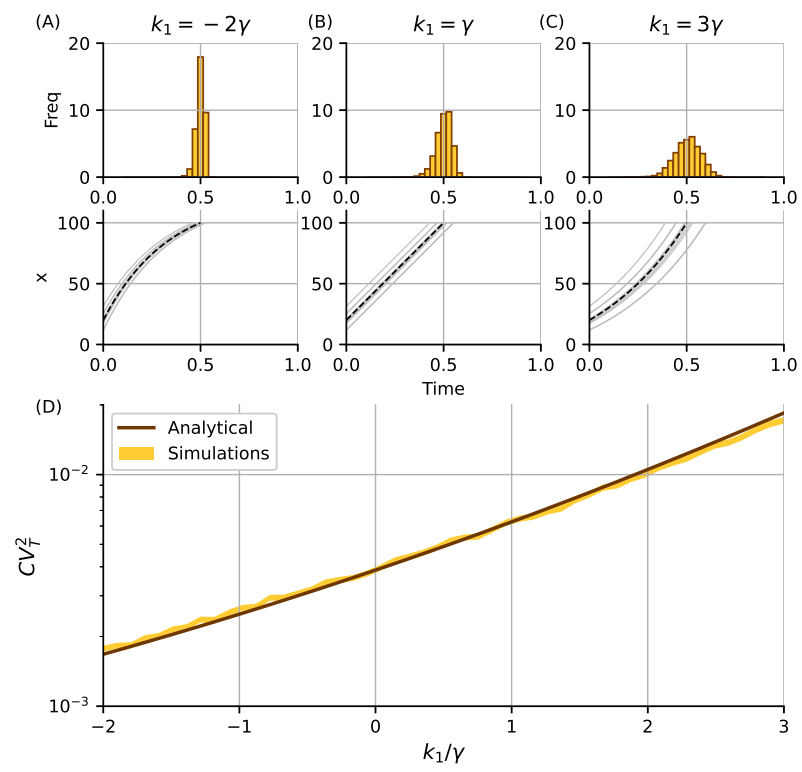

Fig. 4. FPT fluctuations for different feedback strategies considering both random initial concentration $x_{0}$ and deterministic trajectories. (A) $k_{1}<\gamma$ (B) $k_{1}=\gamma$ (C) $k_{1}>\gamma$ Top: FPT histogram. Bottom: Some illustrative trajectories. (D) Noise in FPT measured by the squared coefficient on variation $C V_{T}^{2}$ vs the feedback strength $k_{1}$ relative to the decay rate $\gamma$. Results of simulations (region width representing 95\% confidence interval for ten thousand simulation replicas) are compared to the analytical expression (34). $\left(\gamma=1,\langle T\rangle \approx 0.5, X=100, x_{0}\right.$ is Gammadistributed with parameters: $\left.\left\langle x_{0}\right\rangle=20, C V_{x_{0}}^{2}=0.1\right)$.

Having set up the mean concentration dynamics we now investigate its variance by taking $m=2$ in (13)

$$
\frac{d\left\langle x^{2}\right\rangle}{d t}=\frac{\left\langle B^{2}\right\rangle}{\langle B\rangle}\left(k_{0}+k_{1}\langle x\rangle\right)+2 k_{0}\langle x\rangle+2 k_{1}\left\langle x^{2}\right\rangle-2 \gamma\left\langle x^{2}\right\rangle .
$$

A geometric approach of connecting the variance in protein concentrations to the variance of threshold-crossing times was suggested in [64]. In particular, the variance in $T$ is approximated as

$$
\sigma_{T}^{2} \approx \lim _{t \rightarrow\langle T\rangle} \frac{\left\langle x^{2}\right\rangle-\langle x\rangle^{2}}{\left(\frac{d\langle x\rangle}{d t}\right)^{2}}
$$

and is inversely proportional to the slope of the mean trajectory at $t=\langle T\rangle$ with a "flatter" approach to the threshold amplifying noise in threshold-hitting times. Substituting the solutions of (14a) and (17) in (18) results in the following analytical expression for the noise in $T$ as quantified by its coefficient of variation

$$
\begin{aligned}
C V_{T}^{2}:=\frac{\sigma_{T}^{2}}{\langle T\rangle^{2}} \approx & \frac{\left\langle B^{2}\right\rangle}{\langle B\rangle} \times\left(\gamma e^{\gamma\langle T\rangle}+e^{k_{1}\langle T\rangle}\left(\gamma-2 k_{1}\right)\right) \times \\
& \frac{e^{-\left(\gamma+2 k_{1}\right)\langle T\rangle}\left(e^{\gamma\langle T\rangle}-e^{k_{1}\langle T\rangle}\right)^{2}}{2\langle T\rangle^{2}\left(\gamma-k_{1}\right)^{3} X} .
\end{aligned}
$$

In the limit of no dilution and no feedback $(\gamma \rightarrow 0$ and $k_{1} \rightarrow 0$ ), the formulas (15) and (19) become exact and reduce 

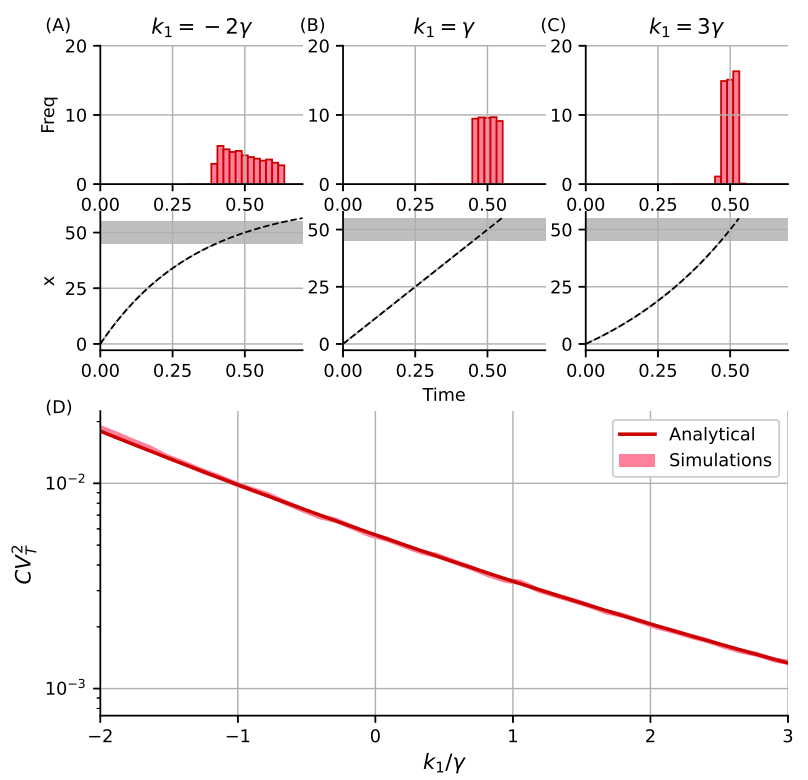

Fig. 5. FPT fluctuations for different feedback strategies considering both random threshold concentration $X$ and deterministic trajectories. (A) $k_{1}<\gamma$ (B) $k_{1}=\gamma$ (C) $k_{1}>\gamma$ Top: FPT histogram. Bottom: Some illustrative trajectories. (D) Noise in FPT measured by the squared coefficient on variation $C V_{T}^{2}$ vs the feedback strength $k_{1}$ relative to the decay rate $\gamma$. Results of simulations (region width representing 95\% confidence interval for ten thousand simulation replicas) are compared to the analytical expression (35). $\left(\gamma=1,\langle T\rangle \approx 0.5, x_{0}=0, X\right.$ is uniformly distributed $U(45,55)$ such as $\langle X\rangle=50$ and $\left.C V_{X}^{2}=1 / 300\right)$.

to

$$
\langle T\rangle=\frac{X}{k_{0}}, \quad C V_{T}^{2}=\frac{\left\langle B^{2}\right\rangle}{\langle B\rangle X} .
$$

Fig. 2 illustrates the shape of $C V_{T}^{2}$ as a function of the feedback strength $k_{1}$, while correspondingly changing $k_{0}$ as per (16) to ensure a fixed $\langle T\rangle$. Two distinct observations can be made from this plot:

- The noise in $T$ as predicted by (19) matches well with corresponding FPT noise levels obtained from stochastic simulation runs of the system (2). As expected, the match is perfect at low noise levels and begins to deviate with increasing $C V_{T}$.

- $C V_{T}$ first decreases with increasing $k_{1}$ to reach a minimum, and then increases with increasing $k_{1}$.

By solving the equation

$$
\frac{d C V_{T}^{2}}{d k_{1}}=0
$$

our analysis predicts the following optimal feedback strength

$$
k_{1} \approx \gamma\left(1+\frac{3}{2+3 \gamma\langle T\rangle}\right)
$$

that minimizes the fluctuations in the threshold-crossing times around $\langle T\rangle$. Notice this optimal solution corresponds to a positive feedback $k_{1}>0$ and depends on the dimensionless factor $\gamma\langle T\rangle$. If $\gamma$ is the exponential growth in cell volume within a cell cycle, then $\gamma\langle T\rangle$ can be interpreted as mean FPT normalised by the cell-cycle duration. In the limit, $\gamma \rightarrow 0$ a no feedback strategy emerges optimal. Moreover, the optimal feedback strength $k_{1} \approx 5 \gamma / 2$ for $\gamma\langle T\rangle \approx 0$ (i.e., the mean FPT is much shorter compared to the cell-cycle duration), $k_{1} \approx 8 \gamma / 3$ for $\gamma\langle T\rangle=1$ and $k_{1} \approx \gamma$ for $\gamma\langle T\rangle \gg 1$.

Does this optimal feedback strategy depend on how noise is incorporated into the model? To address these questions, we consider a simple stochastic differential equation (SDE) formulation

$$
d x(t)=k_{0}+k_{1} x(t)-\gamma x(t)+\sigma d w(t)
$$

where $w(t)$ denotes a Wiener process. Note that the mean dynamics $\langle x(t)\rangle$ in (23) is identical to that of the previous model and given by (14a), but now the variance evolves as

$$
\frac{d\left\langle x^{2}\right\rangle}{d t}=\sigma^{2}+2 k_{0}\langle x\rangle+2 k_{1}\left\langle x^{2}\right\rangle-2 \gamma\left\langle x^{2}\right\rangle .
$$

Performing the exact same analysis as before by substituting $\langle x(t)\rangle$ and the solution of (24) in (18) yields

$$
C V_{T}^{2}:=\frac{\sigma^{2}\left(\operatorname{Sinh}\left[2\langle T\rangle\left(\gamma-k_{1}\right)\right]-2 \operatorname{Sinh}\left[\langle T\rangle\left(\gamma-k_{1}\right]\right)\right.}{\langle T\rangle^{2}\left(\gamma-k_{1}\right)^{3} X^{2}}
$$

for the SDE model. While the qualitative trend of $C V_{T}$ varying non-monotonically with respect to $k_{1}$ is also seen here (Fig. 3), in contrast to the earlier results, the noise in FPT is always minimized at $k_{1}=\gamma$ in the SDE model. Thus, while positive feedback provides precision in event timing in both formulations, the optimal feedback strength depends on the noise structure.

\section{OPTIMAL FEEDBACK STRATEGY FOR NOISY INITIAL CONDITIONS AND THRESHOLD}

Our analysis in the previous section considers the synthesis of a gene product in random bursts as the predominant source of noise driving fluctuations in threshold-crossing times. We now extend this analysis to scenarios where the protein concentrations start from a non-zero initial condition $x_{0}$ and build over time to the threshold $X$, where both $x_{0}$ and $X$ are random variables that are drawn from arbitrary distributions before each simulation run. Based on an analysis similar to (15), the mean FPT conditioned on $x_{0}$ and $X$ is given by

$$
\left\langle T \mid x_{0}, X\right\rangle \approx \frac{\ln \left[\frac{k_{0}+\left(k_{1}-\gamma\right) x_{0}}{k_{0}+\left(k_{1}-\gamma\right) X}\right]}{\gamma-k_{1}} .
$$

In the case of no feedback and no decay $\left(\gamma \rightarrow 0\right.$ and $k_{1} \rightarrow 0$ ), this reduces to

$$
\left\langle T \mid x_{0}, X\right\rangle=\frac{X-x_{0}}{k_{0}},
$$

where the initial condition gets absorbed (subtracted) in the threshold. Assuming small variations in $x_{0}$ and $X$ around their respective means, $\left\langle x_{0}\right\rangle$ and $\langle X\rangle$ results in the mean FPT

$$
\langle T\rangle \approx \frac{\ln \left[\frac{k_{0}+\left(k_{1}-\gamma\right)\left\langle x_{0}\right\rangle}{k_{0}+\left(k_{1}-\gamma\right)\langle X\rangle}\right]}{\gamma-k_{1}}
$$


Solving this equation for $k_{0}$

$$
k_{0}=\frac{\left(k_{1}-\gamma\right)\left(\langle X\rangle-\left\langle x_{0}\right\rangle e^{\left(k_{1}-\gamma\right)\langle T\rangle}\right)}{e^{\left(k_{1}-\gamma\right)\langle T\rangle}-1}
$$

provides the corresponding changes in $k_{0}$ needed to ensure a given mean FPT as we alter feedback strategies.

Our assumption of small fluctuations in $x_{0}$ and $X$ allows us to Taylor expand (26) around their respective means

$$
\frac{\left\langle T \mid x_{0}, X\right\rangle-\langle T\rangle}{\langle T\rangle} \approx 1+S_{X}^{T} \frac{(X-\langle X\rangle)}{\langle X\rangle}+S_{x_{0}}^{T} \frac{\left(x_{0}-\left\langle x_{0}\right\rangle\right)}{\left\langle x_{0}\right\rangle}
$$

where

$$
\begin{aligned}
S_{X}^{T} & =\frac{\langle X\rangle}{\langle T\rangle} \frac{\partial\langle T\rangle}{\partial\langle X\rangle} \\
S_{x_{0}}^{T} & =\frac{\left\langle x_{0}\right\rangle}{\langle T\rangle} \frac{\partial\langle T\rangle}{\partial\left\langle x_{0}\right\rangle}
\end{aligned}
$$

are the dimensionless log-sensitivities of $\langle T\rangle$ with respect to $\left\langle x_{0}\right\rangle$ and $\langle X\rangle$, respectively. Squaring both sides and taking the expectation with respect to $x_{0}$ and $X$ yields the following noise in the FPTs

$$
\begin{aligned}
C V_{T}^{2} & =\left(S_{X}^{T}\right)^{2} C V_{X}^{2}+\left(S_{x_{0}}^{T}\right)^{2} C V_{x_{0}}^{2} \\
\left(S_{x_{0}}^{T}\right)^{2} & =\frac{\left(e^{\left(k_{1}-\gamma\right)\langle T\rangle}-1\right)^{2}\left\langle x_{0}\right\rangle^{2}}{\left(\gamma-k_{1}\right)^{2}\left(\langle X\rangle-\left\langle x_{0}\right\rangle\right)^{2}\langle T\rangle^{2}} \\
\left(S_{X}^{T}\right)^{2} & =\frac{\left(e^{\left(\gamma-k_{1}\right)\langle T\rangle}-1\right)^{2}\langle X\rangle^{2}}{\left(\gamma-k_{1}\right)^{2}\left(\langle X\rangle-\left\langle x_{0}\right\rangle\right)^{2}\langle T\rangle^{2}}
\end{aligned}
$$

with $C V_{x_{0}}$ and $C V_{X}$ being the coefficient of variation of the initial condition and threshold. Both sensitivities look similar in form but they do differ from each other in the sign of $\gamma-k_{1}$ in the numerator that makes a qualitative difference - while $\left(S_{X}^{T}\right)^{2}$ is a decreasing function of $k_{1}$ with $\left(S_{X}^{T}\right)^{2} \rightarrow 0$ as $k_{1} \rightarrow \infty,\left(S_{x_{0}}^{T}\right)^{2}$ is an increasing function of $k_{1}$ and $\left(S_{x_{0}}^{T}\right)^{2} \rightarrow 0$ as $k_{1} \rightarrow-\infty$. An important conclusion from this is that in the presence of noisy initial conditions, choosing $k_{1}$ to be as negative as possible (strong negative feedback) is the optimal strategy to minimize $C V_{T}^{2}$ around a given $\langle T\rangle$. In contrast, for a noisy threshold choosing $k_{1}$ to be as positive as possible (strong positive feedback) is the optimal strategy. We illustrate these results in Fig. 4 (a fixed threshold and a noisy initial condition) and Fig. 5 (a zero initial condition and a noisy threshold), where analytically predicted $C V_{T}^{2}$ match well with those obtained from simulations confirming our predictions for different forms of feedback.

\section{CONCLUSION}

Uncovering mechanisms regulating the precise temporal triggering of events is vital for diverse cellular processes from development to cell-cycle regulation [1]-[8]. This contribution has explored feedback strategies that buffer fluctuations in the first-passage time around a given mean event time. To provide some biological context to this problem, consider $E$. coli cells infected by the virus bacteriophage lambda, where lysis of individual cells is the result of expression and accumulation of a single viral protein (holin) in the bacterial cell membrane up to a critical threshold [11], [12], [31], [35]. Since there is an optimal time to lyse the cells [65]-[67], the holin accumulation needs to occur to reach the threshold at the optimal lysis time. Indeed, recent experiments show that faster or slower lysis than this optimal time can result in significant fitness defects for the virus.

Using a geometric approach that connects the variance in protein concentrations to the variance in the thresholdcrossing times, we derived analytical expressions for the noise in FPT's assuming linear feedback regulation of gene expression. Our results show good agreement with Monte Carlo simulations (Figs. 2 and 3) and determined the optimal positive feedback strength needed for precision in timing. This feedback strength decreases with decreasing dilution rate and converges to a no-feedback strategy in the case of no protein decay. An SDE formulation of the problem with identical dynamics for the mean protein concentration resulted in a different positive feedback strength suggesting that this value is dependent on how noise is formulated in the model. Finally, we also considered cell-to-cell variation in FPT arising from noise in initial conditions and timing threshold in which case a strong negative or positive feedback is needed, respectively, to minimize the FPT fluctuations

As part of future work, we will consider other known sources of stochasticity, such as noise arising in the partitioning of molecules during cell division [68], [69], and extrinsic fluctuations in the growth of cell size that would be reflected in the dilution rate [70], [71]. While this work restricts the analysis to linear feedback, we will also consider Hill function type nonlinear feedback in the future, where we can explore optimal strategies by employing a combination of approximate closure schemes [62], [72], [73] and stochastic simulations.

\section{ACKNOWLEDGMENT}

\section{AS is supported by NIH 1R01GM124446-01.}

\section{REFERENCES}

[1] S. Patra and D. Chowdhury, "Level crossing statistics in a biologically motivated model of a long dynamic protrusion: passage times, random and extreme excursions," Journal of Statistical Mechanics: Theory and Experiment, vol. 2021, no. 8, p. 083207, 2021.

[2] C. R. Gliech and A. J. Holland, "Keeping track of time: the fundamentals of cellular clocks," Journal of Cell Biology, vol. 219, no. 11, 2020.

[3] J. Gerardin, N. R. Reddy, and W. A. Lim, "The design principles of biochemical timers: circuits that discriminate between transient and sustained stimulation," Cell systems, vol. 9, no. 3, pp. 297-308, 2019.

[4] J. Qian, L. Gelens, and M. Bollen, "Coordination of timers and sensors in cell signaling," BioEssays, vol. 41, no. 3, p. 1800217, 2019.

[5] M. Zhu, W. Chen, V. Mirabet, L. Hong, S. Bovio, S. Strauss, E. M. Schwarz, S. Tsugawa, Z. Wang, R. S. Smith, et al., "Robust organ size requires robust timing of initiation orchestrated by focused auxin and cytokinin signalling," Nature Plants, pp. 1-13, 2020.

[6] A. T. Winfree, The geometry of biological time, vol. 12. Springer Science \& Business Media, 2001.

[7] L. Rensing, U. Meyer-Grahle, and P. Ruoff, "Biological timing and the clock metaphor: oscillatory and hourglass mechanisms," Chronobiology international, vol. 18, pp. 329-369, 2001.

[8] J. M. Pedraza and J. Paulsson, "Random timing in signaling cascades," Molecular Systems Biology, vol. 3, p. 81, 2007. 
[9] A. Zaslaver, A. E. Mayo, R. Rosenberg, P. Bashkin, H. Sberro, M. Tsalyuk, M. G. Surette, and U. Alon, "Just-in-time transcription program in metabolic pathways," Nature Genetics, vol. 36, pp. 486491, 2004

[10] N. Friedman, S. Vardi, M. Ronen, U. Alon, and J. Stavans, "Precise temporal modulation in the response of the SOS DNA repair network in individual bacteria," PLoS biology, vol. 3, no. 7, p. e238, 2005.

[11] J. J. Dennehy and I.-N. Wang, "Factors influencing lysis time stochasticity in bacteriophage $\lambda$," BMC Microbiology, vol. 11, p. 174, 2011.

[12] R. White, S. Chiba, T. Pang, J. S. Dewey, C. G. Savva, A. Holzenburg, K. Pogliano, and R. Young, "Holin triggering in real time," Proceedings of the National Academy of Sciences, vol. 108, pp. 798-803, 2011.

[13] S. Kannoly, T. Gao, S. Dey, N. Wang, A. Singh, and J. J. Dennehy, "Optimum threshold minimizes noise in timing of intracellular events," iScience, vol. 23, p. 101186, 2020.

[14] C. Schwarz, A. Johnson, M. Kõivomägi, E. Zatulovskiy, C. J. Kravitz, A. Doncic, and J. M. Skotheim, "A precise Cdk activity threshold determines passage through the restriction point," Molecular cell, vol. 69, no. 2, pp. 253-264, 2018.

[15] Y. Goldschmidt, E. Yurkovsky, A. Reif, R. Rosner, A. Akiva, and I. Nachman, "Control of relative timing and stoichiometry by a master regulator," PLOS ONE, vol. 10, pp. 1-14, 2015.

[16] I. Nachman, A. Regev, and S. Ramanathan, "Dissecting timing variability in yeast meiosis," Cell, vol. 131, pp. 544-556, 2007.

[17] E. Yurkovsky and I. Nachman, "Event timing at the single-cell level," Briefings in Functional Genomics, vol. 12, pp. 90-98, 2013.

[18] K. Carniol, P. Eichenberger, and R. Losick, "A threshold mechanism governing activation of the developmental regulatory protein $\sigma \mathrm{F}$ in Bacillus subtilis," Journal of Biological Chemistry, vol. 279, pp. 14860-14870, 2004.

[19] S. L. Spencer, S. Gaudet, J. G. Albeck, J. M. Burke, and P. K. Sorger, "Non-genetic origins of cell-to-cell variability in TRAILinduced apoptosis," Nature, vol. 459, pp. 428-432, 2009.

[20] J. Roux, M. Hafner, S. Bandara, J. J. Sims, H. Hudson, D. Chai, and P. K. Sorger, "Fractional killing arises from cell-to-cell variability in overcoming a caspase activity threshold," Molecular Systems Biology, vol. 11, p. 803, 2015.

[21] A. L. Paek, J. C. Liu, A. Loewer, W. C. Forrester, and G. Lahav, "Cell-to-cell variation in p53 dynamics leads to fractional killing," Cell, vol. 165, no. 3, pp. 631-642, 2016.

[22] Y.-Y. Cheng, A. J. Hirning, K. Josic, and M. R. Bennett, "The timing of transcriptional regulation in synthetic gene circuits," ACS synthetic biology, vol. 6, no. 11, pp. 1996-2002, 2017.

[23] L. Cai and N. F. X. S. Xie, "Stochastic protein expression in individual cells at the single molecule level," Nature, vol. 440, pp. 358-362, Sept. 2006.

[24] J. M. Raser and E. K. O'Shea, "Noise in gene expression: Origins, consequences, and control," Science, vol. 309, pp. 2010 - 2013, 2005.

[25] A. Raj and A. van Oudenaarden, "Nature, nurture, or chance: stochastic gene expression and its consequences," Cell, vol. 135, pp. 216-226, 2008.

[26] M. Kærn, T. C. Elston, W. J. Blake, and J. J. Collins, "Stochasticity in gene expression: from theories to phenotypes," Nature Reviews Genetics, vol. 6, pp. 451-464, 2005.

[27] A. M. Corrigan, E. Tunnacliffe, D. Cannon, and J. R. Chubb, "A continuum model of transcriptional bursting," eLife, vol. 5, p. e13051, 2016.

[28] R. D. Dar, B. S. Razooky, A. Singh, T. V. Trimeloni, J. M. McCollum, C. D. Cox, M. L. Simpson, and L. S. Weinberger, "Transcriptional burst frequency and burst size are equally modulated across the human genome," Proceedings of the National Academy of Sciences, vol. 109, pp. 17454-17459, 2012.

[29] T. Lu, T. Shen, C. Zong, J. Hasty, and P. G. Wolynes, "Statistics of cellular signal transduction as a race to the nucleus by multiple random walkers in compartment/phosphorylation space," Proceedings of the National Academy of Sciences, vol. 103, no. 45, pp. 16752-16757, 2006.

[30] R. Murugan and G. Kreiman, "On the minimization of fluctuations in the response times of autoregulatory gene networks," Biophysical journal, vol. 101, pp. 1297-1306, 2011.

[31] A. Singh and J. J. Dennehy, "Stochastic holin expression can account for lysis time variation in the bacteriophage $\lambda$," Journal of the Royal Society Interface, vol. 11, p. 20140140, 2014.
[32] K. R. Ghusinga and A. Singh, "Theoretical predictions on the firstpassage time for a gene expression model," in 2015 54th IEEE Conference on Decision and Control (CDC), pp. 3864-3869, IEEE, 2015.

[33] M. Shreshtha, A. Surendran, and A. Ghosh, "Estimation of mean first passage time for bursty gene expression," Physical biology, vol. 13, no. 3, p. 036004, 2016.

[34] M. C. Lagomarsino, M. Caselle, M. Osella, et al., "Stochastic timing in gene expression for simple regulatory strategies," Nucleic Acids Research, p. gkw1235, 2016.

[35] K. R. Ghusinga, J. J. Dennehy, and A. Singh, "First-passage time approach to controlling noise in the timing of intracellular events," Proceedings of the National Academy of Sciences, vol. 114, pp. 693698, 2017.

[36] S. Gupta, J. Varennes, H. C. Korswagen, and A. Mugler, "Temporal precision of regulated gene expression," PLoS computational biology, vol. 14, p. e1006201, 2018.

[37] K. Biswas, M. Shreshtha, A. Surendran, and A. Ghosh, "First-passage time statistics of stochastic transcription process for time-dependent reaction rates," The European Physical Journal E, vol. 42, no. 2, pp. 111, 2019.

[38] K. Rijal, A. Prasad, and D. Das, "Protein hourglass: Exact first passage time distributions for protein thresholds," Physical Review E, vol. 102, no. 5, p. 052413, 2020.

[39] S. Gupta, S. Fancher, H. C. Korswagen, and A. Mugler, "Temporal precision of molecular events with regulation and feedback," Physical Review E, vol. 101, no. 6, p. 062420, 2020.

[40] K. Biswas, M. K. Jolly, and A. Ghosh, "First passage time properties of miRNA-mediated protein translation," Journal of Theoretical Biology, vol. 529, p. 110863, 2021.

[41] W. Y. C. Huang, S. Alvarez, Y. Kondo, J. Kuriyan, and J. T. Groves, "Relating cellular signaling timescales to single-molecule kinetics: A first-passage time analysis of Ras activation by SOS," Proceedings of the National Academy of Sciences, vol. 118, no. 45, 2021.

[42] M. Cao, B. Qiu, T. Zhou, and J. Zhang, "Control strategies for the timing of intracellular events," Physical Review E, vol. 100, no. 6, p. 062401, 2019.

[43] Z. Vahdat, K. R. Ghusinga, and A. Singh, "Comparing feedback strategies for minimizing noise in gene expression event timing," in 2021 29th Mediterranean Conference on Control and Automation (MED), pp. 450-455, 2021.

[44] K. R. Ghusinga and A. Singh, "Controlling event timing precision for gene expression with external disturbances," in 2020 59th IEEE Conference on Decision and Control (CDC), pp. 5867-5872, 2020.

[45] K. R. Ghusinga and A. Singh, "Effect of gene-expression bursts on stochastic timing of cellular events," in 2017 American Control Conference (ACC), pp. 2118-2123, IEEE, 2017.

[46] K. R. Ghusinga and A. Singh, "Regulating gene expression to achieve temporal precision," IFAC-PapersOnLine, vol. 54, no. 9, pp. 502506, 2021. 24th International Symposium on Mathematical Theory of Networks and Systems MTNS 2020.

[47] S. Dey, S. Kannoly, P. Bokes, J. J. Dennehy, and A. Singh, "The role of incoherent feedforward circuits in regulating precision of event timing," bioRxiv, 2020.

[48] K. Rijal, A. Prasad, A. Singh, and D. Das, "Exact distribution of threshold crossing times for protein concentrations: Implication for biological timekeeping," Phys. Rev. Lett., vol. 128, p. 048101, 2022.

[49] O. Padovan-Merhar, G. P. Nair, A. G. Biaesch, A. Mayer, S. Scarfone, S. W. Foley, A. R. Wu, L. S. Churchman, A. Singh, and A. Raj, "Single mammalian cells compensate for differences in cellular volume and DNA copy number through independent global transcriptional mechanisms," Molecular Cell, vol. 58, pp. 339-352, 2015.

[50] K. R. Ghusinga, C. A. Vargas-Garcia, and A. Singh, "A mechanistic stochastic framework for regulating bacterial cell division," Scientific Reports, p. 30229, 2016.

[51] C. A. Vargas-Garcia, K. R. Ghusinga, and A. Singh, "Cell size control and gene expression homeostasis in single-cells," Current opinion in systems biology, vol. 8, pp. 109-116, 2018.

[52] J. Lin and A. Amir, "Homeostasis of protein and mrna concentrations in growing cells," Nature communications, vol. 9, no. 1, pp. 1-11, 2018.

[53] V. Shahrezaei and P. S. Swain, "Analytical distributions for stochastic gene expression," Proceedings of the National Academy of Sciences, vol. 105, pp. 17256-17261, 2008. 
[54] A. Schwabe, K. N. Rybakova, and F. J. Bruggeman, "Transcription stochasticity of complex gene regulation models," Biophysical Journal, vol. 103, no. 6, pp. 1152-1161, 2012.

[55] H. Kuwahara, S. T. Arold, and X. Gao, "Beyond initiation-limited translational bursting: the effects of burst size distributions on the stability of gene expression," Integrative Biology, vol. 7, no. 12, pp. 1622-1632, 2015.

[56] N. Kumar, A. Singh, and R. V. Kulkarni, "Transcriptional bursting in gene expression: analytical results for general stochastic models," PLoS computational biology, vol. 11, no. 10, p. e1004292, 2015.

[57] D. A. McQuarrie, "Stochastic approach to chemical kinetics," Journal of applied probability, vol. 4, pp. 413-478, 1967.

[58] N. G. Van Kampen, Stochastic processes in physics and chemistry, vol. 1. Elsevier, 1992.

[59] D. T. Gillespie, "A general method for numerically simulating the stochastic time evolution of coupled chemical reactions," Journal of computational physics, vol. 22, no. 4, pp. 403-434, 1976.

[60] X. Mao, "The truncated euler-maruyama method for stochastic differential equations," Journal of Computational and Applied Mathematics, vol. 290, pp. 370-384, 2015.

[61] J. P. Hespanha and A. Singh, "Stochastic models for chemically reacting systems using polynomial stochastic hybrid systems," International Journal of Robust and Nonlinear Control, vol. 15, pp. 669-689, 2005.

[62] A. Singh and J. P. Hespanha, "Approximate moment dynamics for chemically reacting systems," IEEE Transactions on Automatic Control, vol. 56, pp. 414-418, 2011.

[63] A. Singh and J. P. Hespanha, "Stochastic hybrid systems for studying biochemical processes," Philosophical Transactions of the Royal Society A, vol. 368, pp. 4995-5011, 2010.

[64] M. C. Lagomarsino, M. Caselle, M. Osella, et al., "Stochastic timing in gene expression for simple regulatory strategies," Nucleic Acids Research, p. gkw1235, 2016.
[65] R. H. Heineman and J. J. Bull, "Testing optimality with experimental evolution: lysis time in a bacteriophage," Evolution, vol. 61, pp. 16951709, 2007.

[66] J. A. Bonachela and S. A. Levin, "Evolutionary comparison between viral lysis rate and latent period," Journal of Theoretical Biology, vol. 345, pp. 32-42, 2014.

[67] S. Kannoly, A. Singh, and J. J. Dennehy, "An optimal lysis time maximizes bacteriophage fitness in quasi-continuous culture," bioRxiv, pp. 2020-05, 2021.

[68] D. Huh and J. Paulsson, "Random partitioning of molecules at cell division," Proceedings of the National Academy of Sciences, vol. 108, no. 36, pp. 15004-15009, 2011.

[69] M. Soltani, C. A. Vargas-Garcia, D. Antunes, and A. Singh, "Intercellular variability in protein levels from stochastic expression and noisy cell cycle processes," PLoS computational biology, vol. 12, no. 8, p. e1004972, 2016.

[70] C. Cadart, L. Venkova, M. Piel, and M. C. Lagomarsino, "Volume growth in animal cells is cell cycle dependent and shows additive fluctuations," Elife, vol. 11, p. e70816, 2022.

[71] D. Pirjol, F. Jafarpour, and S. Iyer-Biswas, "Phenomenology of stochastic exponential growth," Physical Review E, vol. 95, no. 6, p. 062406, 2017.

[72] K. R. Ghusinga, C. A. Vargas-Garcia, A. Lamperski, and A. Singh, "Exact lower and upper bounds on stationary moments in stochastic biochemical systems," Physical biology, vol. 14, no. 4, p. 04LT01, 2017.

[73] Y. Sakurai and Y. Hori, "Bounding transient moments of stochastic chemical reactions," IEEE control systems letters, vol. 3, no. 2, pp. 290-295, 2018. 